\title{
Evaluation of demand in a rural English hospital emergency department
}

\author{
Paul Turner, Ros Kane, Christine Jackson
}

\section{ABSTRACT}

The purpose of this article is to analyse the patient demand placed on a rural district general hospital (DGH) emergency department within the context of the Purpose, Process, People (PPP) framework used in the private sector. This analysis was undertaken to inform wider evaluation of the implementation of the enterprise culture-the NHS policy to adopt private sector best practice to produce resource use, quality and efficiency improvements. The article concludes with a view that the PPP framework provides methods of calculating the level of discharge necessary to meet the four-hour wait target. Data describing the characteristics and patterns of attending patients can be used to develop an emergency department's processes and people to achieve its time-based target.

\section{Key Words: Emergency care • Health service need • Demand for services}

The enterprise culture is a term defined by Wall and Owen to describe the implementation of health policy evolution towards encouraging improvements to quality and efficiency in the NHS through adoption of private sector best practice methods. The enterprise culture uses performance measurement as a key motivator and evaluation tool (Wall and Owen, 2003: 113-125).

Womack argues that successful enterprise improvements come from a pathway of clear (quantified) purpose, robust processes and capable, empowered people-the Purpose, Process, People (PPP) framework common to innovative enterprises (2011).

Silvester et al (2004) examine the effects that variations in capacity and demand have on NHS waiting times and their performance targets-such as the four-hour wait target for emergency departments. Typical NHS responses to the process issues which lead to patient waits, Silvester et al argue, $\rightarrow$ rough flawed planning to supply capacity (e.g. saff and beds) to meet patient demand patterns (2004).

The PPP framework responses to manage imbalances between capacity and demand and avoid the formation of queues are more centred on understanding demand characteristics and the productivity of capacity (George, 2003; Silvester et al, 2004). The rationale for this study is to gain an understanding of case site demand characteristics and the potential for adoption into the PPP framework. A future articl ewill address the capacity characteristics to evaluate a case site's ability to meet the four-hour wait performance target .

Using data describing patient deman the case site emergency department, together with
Paul Turner

Senior Lecturer, University of Lincoln

Ros Kane Principal Lecturer, University of Lincoln

Christine Jackson Principal Research Fellow, University of Lincoln

Email:P rner@ulh. nhs.uk 


\section{A single case site is studied to gain understanding of wider research addressing the efficacy of the enterprise culture present in an emergency department}

an assessment of the characteristics of enterprise culture found in the emergency department's service, the efficacy of the enterprise culture to meet the central the four-hour wait target in a rural hospital is evaluated.

This article addresses the research question 'what is the nature of the emergency service patients' demand?' The study describes the emergency department attendance characteristics within the context of the importance that private sector best practice places on planning to meet demand (to explore the 'purpose' aspect of PPP).

\section{Methods}

A single case site is studied to gain understanding of wider research addressing the efficacy of the enterprise culture present in an emergency department (see: Turner et al, 2015). Quantitative data recording patient attendances are extracted and presented to identify characteristics to be considered within a PPP framework in this study.

The single case site is studied within the wi 9 ontext of research to test the theory that tesc he enterprise culture has provided a framework for performance improvement in a rural district general hospital (DGH). The study is generalisable to the theory and not a sample of the population o al DGH (Yin, 2014: 21).

The case site serecced is a DGH in a predominately rural county in England. Alternative acute care is provided by five surrounding hospitals ranging between 30 and 45 miles a d the case site. Ethical approval was grant com committees from the DGH trust, University of Lincoln, and National Research Ethics Service in 2012.
A one-year extract of anonymised data (12 months immediately preceding the qualitative study) detailing all attendances is sourced from the DGH emergency department database-extracted by the trust's information services department.

Emergency department demand is analysed from the data detailing all patients attending the department and accessing the service. These attendances are recorded as individual data and summarised to understand the pressures on the emergency department. Condensing a large amount of information into descriptions of location (centre of the data distribution) and spread (variability of the data) helps the user to consider the data in 'a few intelligible numbers' (Campbell et al, 2007: 28).

Although we are not able to capture long-term trends, the analysis represents the case study population and is validated by clinicians and managers from the emergency department. Validation is achieved using a Delphi group to realise consensus and will be discussed in a future paper. This validation assists the study by mitigating against the risks that Yin describes when using archival evidence: researchers must: 'be careful to ascertain the conditions under which [the evidence] was produced as well as its accuracy' (Yin, 2014: 109).

Selecting the population of all attending patients avoids sample error (Campbell et al, 2007: 81). These data are deemed relevant to investigate the theory in the contemporary case study (Yin, 2014: 109-10) by describing the site in context of the ethnography.

\section{Limitations}

These available data are restricted in duration by the lack of access to previous reliable data following system and coding changes at the sit $O$

\section{Emergency department performance within the enterprise culture}

Efficiency and effectiveness through the PPP framework are achieved by designing and implementing processes and people skills to meet a clear and accurately specified customer purpose (Turner et al, 2013). Although we argue 
that the centralised targets introduced through the enterprise culture are not developed within the PPP 'purpose' context of 'precisely the right value for the customer' (Womack 2005: 6), they do present a performance improvement out against which to test this study's theory (to 2 f the enterprise culture has provided a framework for performance improvement in a rural DGH). The emergency department performance target, developed through the enterprise culture under the Department of Health's first reforming emergency care paper, states that patients should not 'wait more than four hours in an [emergency department] from arrival to admission to a bed in the hospital, transfer elsewhere or discharge. The average length of waiting should fall to 75 minutes (D)partment of Health, 2001).

\section{The rate of discharge to meet performance}

Improving time-related delivery through the PPP framework requires an understanding of the demand placed on a service, and the rate at which work is completed. Time taken to deliver work (lead time) can be calculated using Little's Law (George, 2003: 26), where 'work in progress' is the number of items waiting to be completed and the 'completion rate' is the number of items that can be completed in a specified time frame.

Lead time $=\frac{\text { Amount of work in progress }}{\text { Average completion rate }}$

Within an emergency department, this formula would read:

$\begin{aligned} & \text { Time in emergency } \\ & \text { department }\end{aligned}=\frac{\text { Patients in department }}{\text { Average discharge rate }}$

To improve performance in time spent in the emergency department, two options are available: decrease the ients in the department or increase the rate of discharge. Understanding demand is necessary to intervene on both of these options-either through diversion of patients, or improving process or people to increase the completion rate for patient cohorts.

\section{Results of case site analysis Annual patterns}

48919 patients attended the department over the year. The daily attendance figures, in chronological order from 1 April 2011 (data point 1), are shown in Figure 1.

These data show no clear evidence of trend or seasonal patterns. Daily attendances are accepted as normally distributed in the year ( $p=0.2$ in both Kolmogorov-Smirnov and Shapiro-Wilk tests for normality) around a daily mean of 133 patients with a standard deviation of 13.6 patients.

\section{Detailed attendance patterns}

How , analysis of a shorter timeframe shows greater revels of variation. Figure 2 shows the $95 \%$ confidence interval for mean hourly attendances by weekday.

\section{Takt-time concept to indicate productivity}

Adjustments in the service provision across the day-by-hour period, to synchronise the rate of discharge to the demand for service, are necessary to avoid build-up of patients in department (Womack and Jones, 1996: 55-56). The PPP response to this concept is the 'takttime' technique. Takt-time is the average elapsed time between patient discharges-and movement between each treatment step while in department (Womack and Jones, 1996: 55). It is calculated that by taking the time available in a work period and then dividing this by the number of patient attendances. As 'takttimes change when customer demand changes' (Miltenburg, 2007: 356), when calculated, takttime by hour over the week can become a local departmental indicator against performance and investigate improvement potential can be measured.

However, the emergency department has two work-streams which split patient demand: major work-streams (including resuscitation)-higher acuity patients who are likely to be admittedand a minor work-stream. The emergency department definition of patients presenting to the major stream are all arrivals through emergency services and any other patients 


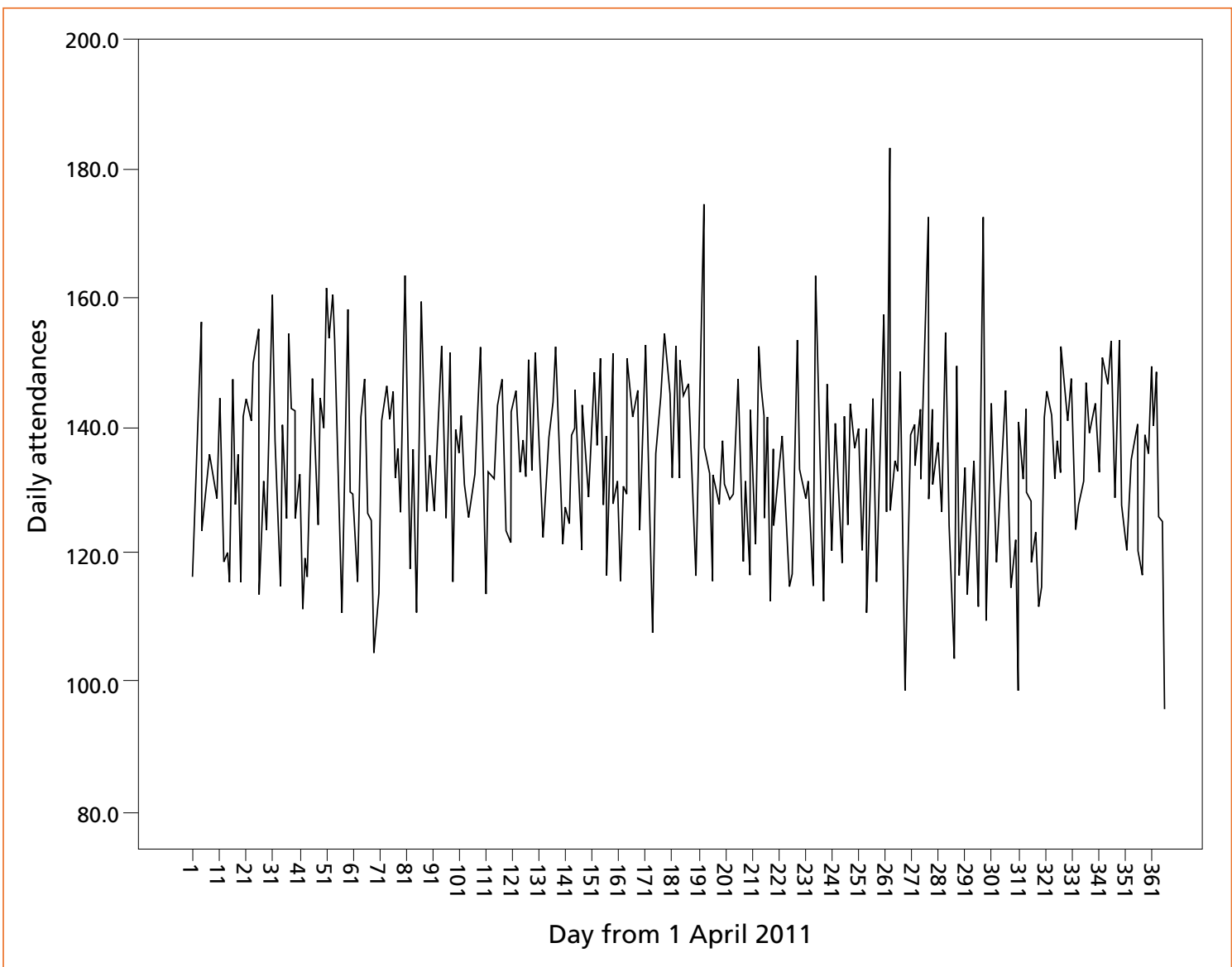

Figure 1. Daily attendances

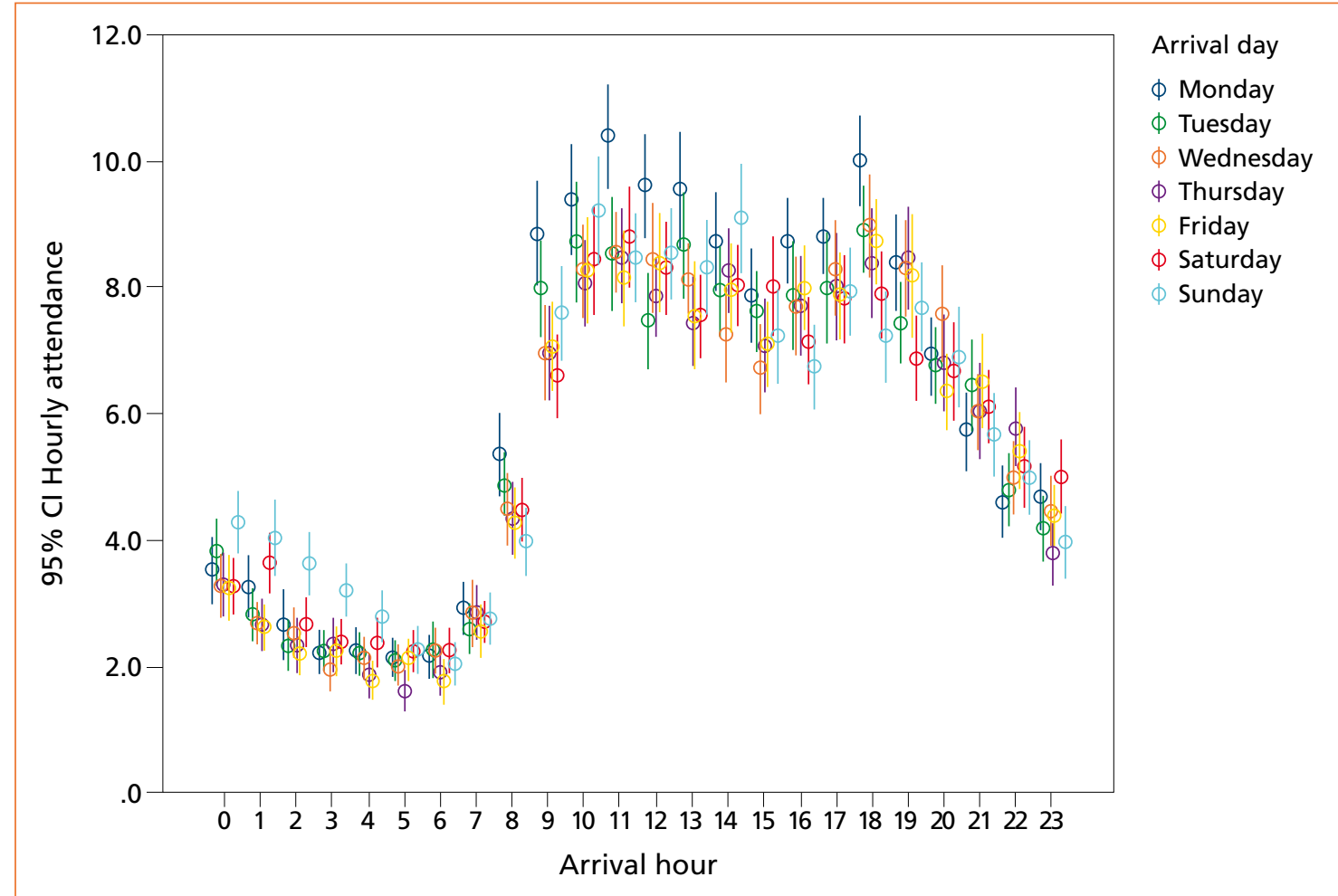

Figure 2. Hourly attendances by day of week (all patients) 


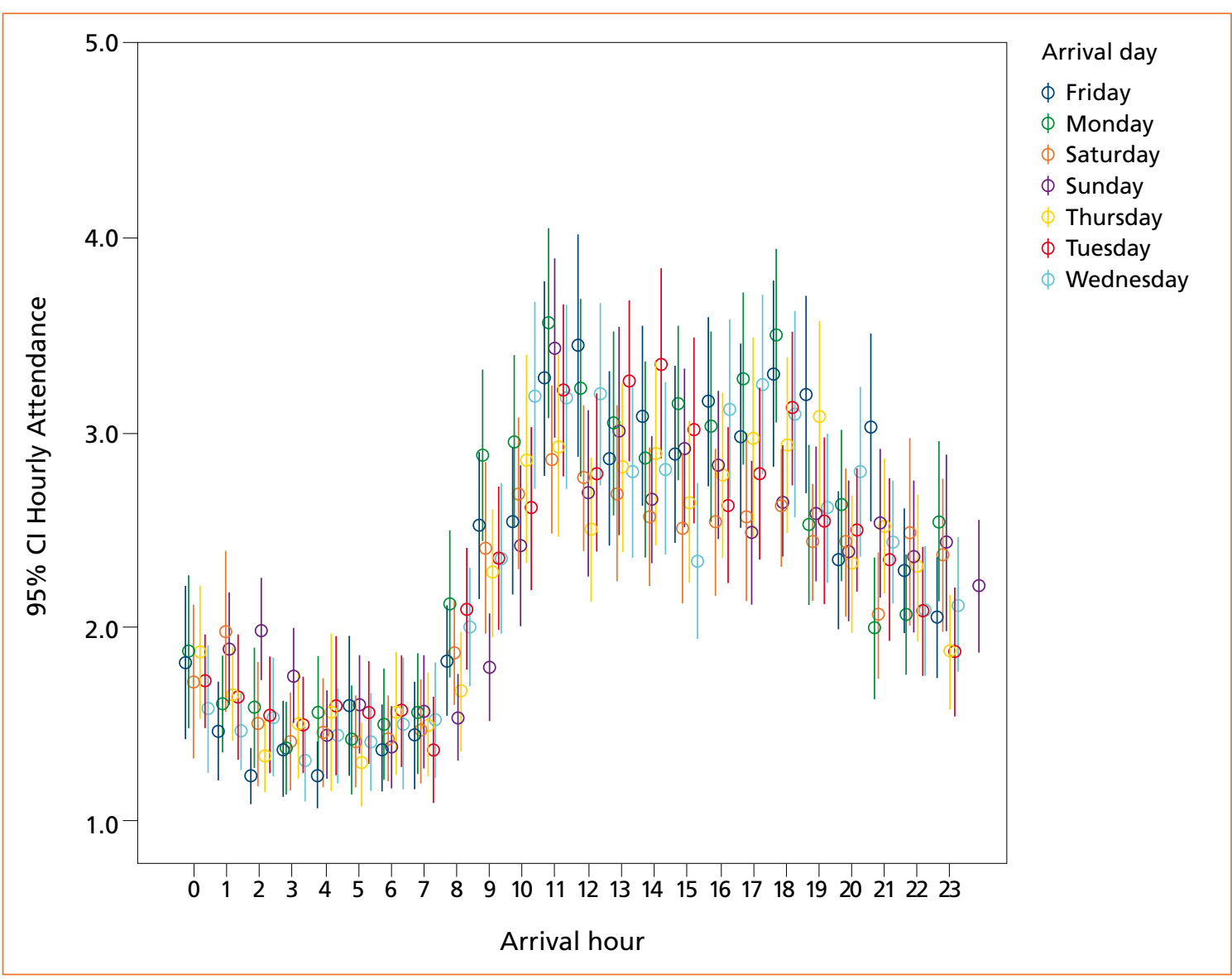

Figure 3a. Hourly attendances by day of week (patients admitted or died in department)

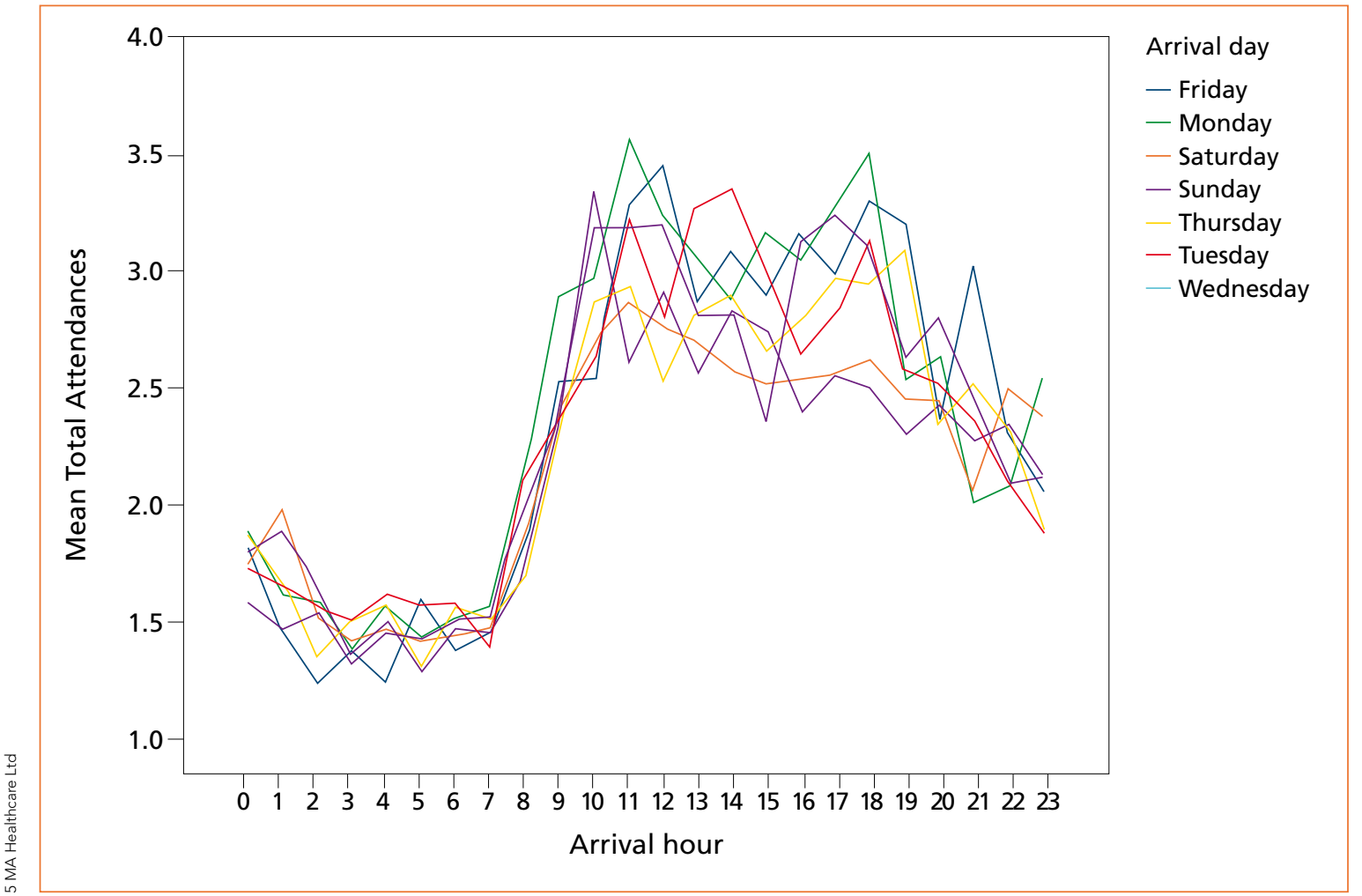

Figure 3 b. Hourly attendances by day of week (patients admitted or died in department) 


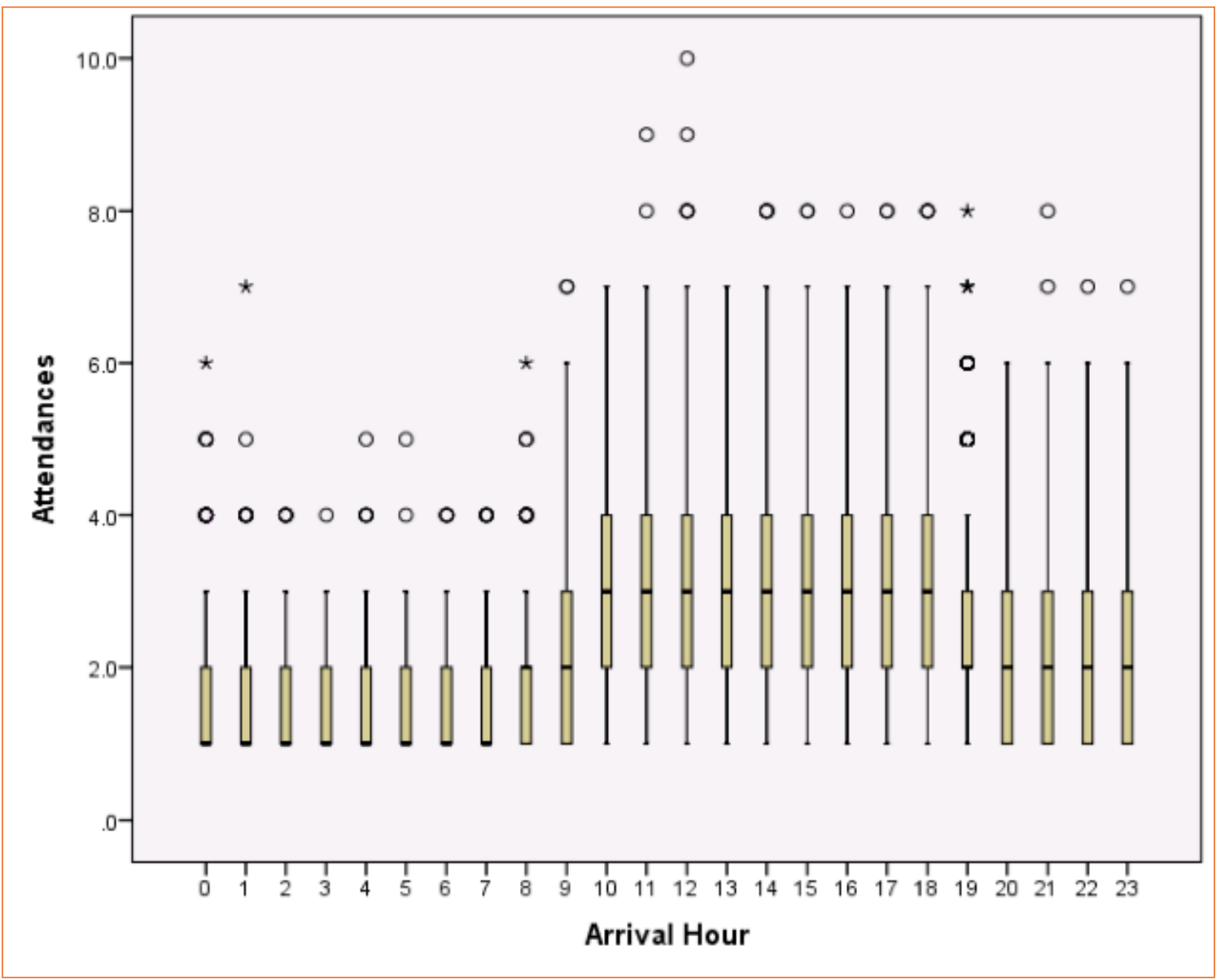

Figure 4. Hourly attendance (patients admitted or died in department)

assessed as such at triage. All other patients are classed as minor. The data extract does not allow analysis using the emergency department's definition of the stream patients were treated in as no such data category is available. However, an analysis of patients admitted as a hospital inpatient or who died in the department is shown in Figure 3 to offer a comparison (these patients are deemed 'major' by the information services department who provided the data).

Figure 3 shows that attendances by week day and hour-of-day follow similar patterns, although a chi-square test comparing the

\section{A constant view of patient arrival is necessary to ensure Miltenburg's flexible takt-times so that discharge productivity can be adjusted when demand varies significantly}

frequency of attendances rejects the hypothesis that the observed number of patients attending by day and hour is consistent with the expected attendances by day and hour $(p=0.001)$.

However, the hourly pattern is consistent between days, an ge of difference between hourly attendances on different days is $t$ more than patien (ए) hour. This analysis can be used as a starting point for calculating the takttime for a 'baseline' day (or days) for patients demanding higher levels of care if greater understanding of the true extent of patien us $\mathrm{ng}$ each workstream was available.

Although there is variation between weekdays, the largest source of uncertainty from the data is the width of the confidence intervals around the mean. When grouped by arrival hour, all test for normality are rejected $(p=0.00$ in both Kolmogorov-Smirnov and Shapiro-Wilk tests for normality for all hours) and each hour fits an exponential distribution. To describe the range 
of data around the median, a boxplot is used in Figure 4.

Although the inter-quartile ranges (vertical oblong boxes) and median (horizontal black line within the inter-quartile range) have a relatively small extent, the data outside this area (particularly the extent over the 75th percentile) extend significantly further. Statistical outliers (marked o or *) also show a variation in the attendance outside of the central tendency.

\section{Discussion}

Through the PPP framework, the amount of time patients wait in an emergency department can be calculated using Little's Law. This would require a dynamic view of the number of patients in the department and an appropriate rate od charges. To ensure that discharge is synchronised to demand patterns, takt-time could be calculated to quantify the productivity needed from the department's processes and people. Such evidence could lead to the production of departmental indicators, which highlight the need for immediate intervention if a problem occurs.

The purpose of analysis through Little's Law and takt-time is to provide an initial profile for matching demand with discharge performance to meet the central target. The range of arrival data and variation described in the case site may present difficulties in planning resources to meet productivity, but knowledge of this level of variation is critical to inform baseline processes and staff planning to provide a flexible service. A constant view of patient arrival is necessary to ensure Miltenburg's flexible takt-times (2007:3556) so that discharge productivity can be adjusted when demand varies significantly (or indeed case-mix deviates from expectation -a level of analysis not possible from this case site data). Their purpose is to provide a clear indication of the rate of productivity to be achieved if the target is to be met and this is an indicator for immediate local action.

This article has shown, through a case study of a rural DGH, a PPP response to the purpose value assigned to an emergency department in a rural DGH emergency care system under the enterprise culture. Although a PPP response to the four-hour wait target cannot be fully calculated from the source data, a framework to achieve the purpose can be planned at a local level.

The next article in this series will review the methods used by the case site emergency department to meet demand and compare to the PPP framework approach in this paper. The article will also discuss the way in which the enterprise culture has provided capacity to meet demand through analysis of pathways and controls and the emergency department resources available. This will be researched using an ethnographic study with validation of the research findings and evaluation of the empirical evidence gathered (the process and people aspect of PPP). BJHCM

\section{References}

Campbell MJ, Machin D, Walters SJ (2007) Medical Statistics: a Textbook for the Health Sciences. 4th edn. Wiley, Chichester

Department of Health (2001) Reforming Emergency Care: first steps to a new approach. Available at: http:// webarchive.nationalarchives.gov.uk/20130107105354/ http://www.dh.gov.uk/prod_consum_dh/groups/ dh_digitalassets/@dh/@en/documents/digitalasset/ dh_4058836.pdf (accessed 28 May 2015)

George ML (2003) Lean Six Sigma for Service: How to Use Lean Speed and Six Sigma Quality to Improve Services and Transactions. McGraw-Hill Education

Miltenburg J (2007) Level schedules for mixed-model JIT production lines: Characteristics of the largest instances that can be solved optimally. International Journal of Production Research 45(16) 3555-577

Silvester K, Lendon R, Bevan H, Steyn R, Walley P (2004) Reducing waiting times in the NHS: Is lack of capacity the problem? Clinician in Management 12(3) 105-9

Turner P, Kane R, Jackson C (2013) Enterprise efficiency framework: The english NHS. BJHCM 19(11): 540-44

Turner P, Kane R, Jackson C (2015) Combining methods to research an emergency department: A case study. BJHCM 21(2): 81-5

Wall A, Owen BG (2003) Health Policy. 2nd edn. Routledge. New York, NY

Womack JP (2005) Going Lean in Health Care. Institute for Healthcare Improvement. Cambridge, MA.

Womack JP (2011) Gemba Walks. Lean Enterprises Institute Inc, Cambridge, MA

Womack JP, Jones DT (1996) Lean Thinking: Banish Waste and Create Wealth in Your Corporation. Simon \& Schuster, London

Yin RK (2014) Case Study Research: Design and Methods. 5th edn. Sage, Thousand Oaks, CA 